\title{
Gravidez na adolescência e o desfecho da prematuridade: uma revisão integrativa de literatura
}

\author{
Pregnancy in adolescence and the prematurity outlet: an integrative literature review \\ Embarazo en adolescencia y entrega de prematuridad: una revisión integradora de la \\ literatura
}

Raquel Vieira Farias ${ }^{1 *}$, Caroline Fernandes Soares e Soares ${ }^{1}$, Rebeca da Silva Araújo ${ }^{1}$, Vivian Ranyelle Soares de Almeida ${ }^{1}$, Dielly de Souza Leitão ${ }^{1}$, Juliana Silva dos Santos ${ }^{1}$, Laisa Silva Santos $^{1}$, Sara Daniele de Araujo Nogueira ${ }^{1}$, Aisiane Cedraz Morais ${ }^{1}$, Caroline Barreto Freire Oliveira ${ }^{1}$.

\section{RESUMO}

Objetivo: Analisar como a produção científica aborda a relação entre a gestação na adolescência com o desfecho da prematuridade. Métodos: Revisão integrativa nas bases de dados Biblioteca Virtual em Saúde (BVS) e Scientific Eletronic Library Online com os descritores Adolescência AND Prematuridade. Trata-se de uma pesquisa vinculada ao Núcleo Interdisciplinar de Estudos de Vulnerabilidade e Saúde da Universidade Estadual de Feira de Santana, na Bahia. A coleta de dados ocorreu entre março e abril de 2020, foram selecionados 2.838 artigos inicialmente e após os critérios de inclusão totalizaram 7 artigos. Resultados: Os artigos revisados apresentaram divergências na abordagem da gravidez precoce com a incidência de prematuridade, evidenciando a influência do pré-natal e do nível de escolaridade no desfecho desse fenômeno. Considerações finais: Os riscos obstétricos e fetais só serão diminuídos quando houver uma rede de apoio direcionada às adolescentes, que foquem na atenção reprodutiva, pré-natal e suporte psicológico e social.

Palavras-chave: Adolescência, Prematuridade, Gestação na adolescência.

\begin{abstract}
Objective: To analyze how scientific production addresses the relationship between pregnancy in adolescence and the outcome of prematurity. Methods: Integrative review of the Virtual Health Library (BVS) and Scientific Eletronic Library Online databases with the descriptors Adolescence AND Prematurity. This is a research linked to the Interdisciplinary Center for Vulnerability and Health Studies at the State University of Feira de Santana, in Bahia. Data collection took place between March and April 2020, 2.838 articles were initially selected and after the inclusion criteria totaled 7 articles. Results: The reviewed articles showed divergences in the approach of early pregnancy with the incidence of prematurity, showing the influence of prenatal care and the level of education on the outcome of this phenomenon. Final considerations: Obstetric and fetal risks will only be reduced when there is a support network aimed at adolescents, who focus on reproductive, prenatal care and psychological and social support.
\end{abstract}

Key words: Adolescence, Prematurity, Pregnancy in adolescence.

1 Universidade Estadual de Feira de Santana (UEFS). Feira de Santana - BA.
${ }^{*}$ E-mail: raquelvieirafariass@gmail.com

SUBMETIDO EM: 5/2020

ACEITO EM: 6/2020

PUBLICADO EM: $\mathbf{8 / 2 0 2 0}$ 


\section{RESUMEN}

Objetivo: analizar cómo la producción científica aborda la relación entre el embarazo en la adolescencia y el resultado de la prematuridad. Métodos: Revisión integral de las bases de datos en línea de la Biblioteca Virtual en Salud (BVS) y la Biblioteca Electrónica Científica con los descriptores Adolescencia AND Prematuridad. Esta es una investigación vinculada al Centro Interdisciplinario de Estudios de Vulnerabilidad y Salud en la Universidad Estatal de Feira de Santana, en Bahía. La recopilación de datos tuvo lugar entre marzo y abril de 2020, inicialmente se seleccionaron 2.838 artículos y luego de los criterios de inclusión totalizaron 7 artículos. Resultados: Los artículos revisados mostraron divergencias en el enfoque del embarazo temprano con la incidencia de prematuridad, mostrando la influencia de la atención prenatal y el nivel de educación sobre el resultado de este fenómeno. Consideraciones finales: los riesgos obstétricos y fetales solo se reducirán cuando haya una red de apoyo dirigida a adolescentes, que se centren en la atención reproductiva, prenatal y el apoyo psicológico y social.

Palabras clave: Adolescencia, Prematuridad, Embarazo en la adolescencia.

\section{INTRODUÇÃO}

A adolescência é uma fase de transição entre a infância e a vida adulta que ocorre de maneira rápida e profunda, onde vários fatores influenciam diretamente na constituição da personalidade desse sujeito. As mesmas autoras relatam diversas transformações físicas, mentais, emocionais, bem como mudanças envolvendo a sexualidade e a descoberta do novo corpo (BUSSMANN CE e PRETTO B, 2017). Dessa forma, é possível compreender que a sexualidade é construída, sendo este momento pertencente ao desenvolvimento da personalidade do adolescente, e todas essas transformações psicossociais interferem no convívio familiar e social, por fazerem parte do processo de formação de sua identidade (PEREIRA FAF, et al., 2017). Assim, ao descobrir e iniciar as práticas sexuais, os adolescentes se tornam mais susceptíveis a infecções sexualmente transmissíveis, conflitos entre casais e gravidez na adolescência (BRASIL, 2017).

O principal fator responsável pela ocorrência da gravidez precoce trata-se da desinformação sobre sexualidade, direitos sexuais e reprodutivos (SBP, 2019). Contudo, outros fatores devem ser considerados, como: clínicos, sociais, culturais, emocionais e também ocorrências de gestações precoces na família, pois muitas vezes as adolescentes grávidas vêm de famílias cujas mulheres engravidaram durante a adolescência (PEREIRA FAF, et al., 2017). Nesse sentido, engravidar nesta faixa etária pode levar a complicações obstétricas como ruptura prematura de membranas, transtorno hipertensivo na gravidez, edema e hemorragia no início da gestação, induzindo a cesariana. Além disso, pode afetar o recém-nascido, causando nascimento prematuro, baixo peso ao nascer, macrossomia e índice de Apgar no primeiro e no quinto minuto menor que sete (TABORDA JA, et al., 2014).

Quanto à prematuridade, por ano cerca de 15 milhões de bebês nascem prematuros, destes 1 milhão vem a óbito e os que sobrevivem apresentam sequelas (CHANG HH, et al., 2013). O nascimento prematuro referese ao parto com idade gestacional superior a $20^{\underline{a}}$ e anterior a $37^{\underline{a}}$ semanas e peso menor de 2.500 gramas (WHO, 2012). Apesar dos indicadores da fecundidade brasileira terem sofrido uma relativa queda nas últimas décadas, de um modo geral, o índice de gravidez na adolescência aumentou.

Conforme CRUZ MS, et al. (2016), a taxa de fecundidade das adolescentes evolui em sentido contrário ao observado para as mulheres de outras faixas etárias, dado que a quantidade de filhos por mulheres vem crescendo nos últimos anos, principalmente em menores de 19 anos de idade, corroborando com o último relatório da Organização das Nações Unidas (ONU, 2020), em que a taxa de fecundidade no Brasil entre meninas de 15 a 19 anos é de 62 a cada mil bebês nascidos vivos, acima da média mundial que é de 44 a cada mil. Assim, essa temática torna-se importante na saúde pública, devido a sua incidência que vem aumentando mundialmente. Ainda de acordo com a pesquisa Nascer Brasil em 2016, 66\% das gestações não são planejadas e cerca de $75 \%$ das mães adolescentes estavam fora da escola, segundo a Pesquisa Nacional por Amostra de Domicílio em 2013, o que pode sugerir o aumento dos índices de abandono da escola, 
mortalidade infantil e pobreza, bem como consequências emocionais e obstétricas, para as mães adolescentes, de modo a impactar no futuro de várias gerações (BRASIL, 2020a).

Além disso, a Organização Pan-Americana da Saúde (OPAS) relata que as adolescentes que engravidam antes de completar 15 anos possuem maior chance de vir a óbito, quando comparadas às adultas, devido a fatores biológicos e socioeconômicos, como imaturidade do sistema reprodutivo, acesso precário aos serviços de saúde, desigualdades raciais/étnicas e pobreza (PAHO, 2018).

Esses dados são significativos e nos remetem à uma reflexão quanto a necessidade de mudanças no planejamento de ações. Um dos mais importantes fatores de prevenção é a discussão sobre sexualidade e saúde reprodutiva, tanto no âmbito familiar e escolar, quanto nos programas de educação em saúde, que devem acompanhar o planejamento reprodutivo e incentivar os adolescentes na busca por orientações sobre as formas de prevenção nas Unidades de Saúde da Atenção Primária, onde há acesso de modo gratuito aos métodos contraceptivos (SBP, 2019).

Diante desse cenário, foi estabelecida a seguinte questão de pesquisa: qual a relação encontrada na literatura entre gestação na adolescência e nascimento de bebês prematuros?

Considerando a alta prevalência de gestação na adolescência, este estudo tem como objetivo analisar como a produção científica aborda a relação entre a gestação na adolescência com o desfecho da prematuridade. São necessárias investigações com a temática, visto que há uma escassez de produções científicas acerca dessa relação direta. Assim, o presente estudo poderá contribuir para elucidar a discussão de como o fator idade se apresenta desfavorável para a saúde materno-infantil.

\section{MÉTODOS}

Trata-se de um estudo do tipo revisão de literatura integrativa, cujo método de pesquisa possibilita a sistematização e análise dos principais resultados de artigos publicados em bancos de dados (RAMALHO NETO JM, et al., 2016). Nessa perspectiva, essa modalidade de revisão permite um acesso rápido aos resultados de pesquisas relevantes, facilitando a difusão do conhecimento (MENDES KDS, et al., 2008).

Para a realização do estudo primeiramente foi estabelecida a questão de pesquisa. De forma ordenada, no período de março a abril de 2020, o levantamento bibliográfico foi executado nas bases de dados Scientific Eletronic Library Online (Scielo) e Biblioteca Virtual em Saúde (BVS), utilizando os descritores "Adolescência AND Prematuridade", totalizando 2.838 textos (Quadro 1).

Quadro 1 - Distribuição dos artigos conforme busca inicial por descritores, 2020.

\begin{tabular}{|c|c|c|}
\hline \multirow{2}{*}{ Processo } & Base de dados & Adolescência AND Prematuridade \\
\hline \multirow{2}{*}{$1^{\text {a }}$ Busca } & Scielo & 37 \\
\cline { 2 - 3 } & BVS & 2.801 \\
\hline \multicolumn{2}{|c|}{ Total } & 2.838 \\
\hline
\end{tabular}

Fonte: Farias RV, et al., 2020.

Em seguida, definiram-se os seguintes critérios de inclusão (filtros): artigos que possuíssem versão completa, no idioma português, publicados entre os anos 2014 a 2019. Foram excluídas dissertações, teses, relatos de experiência, revisões de literatura e artigos que apareceram mais de uma vez na busca.

Totalizando 48 textos. $O$ estudo procedeu com a leitura dos títulos e resumos dos artigos, com a finalidade de selecionar apenas os que possuíssem relação com o tema estudado. Totalizaram 2 artigos no Scielo e 5 na BVS (Figura 1). 
Figura 1 - Fluxograma de identificação e seleção dos artigos para revisão integrativa, 2020.

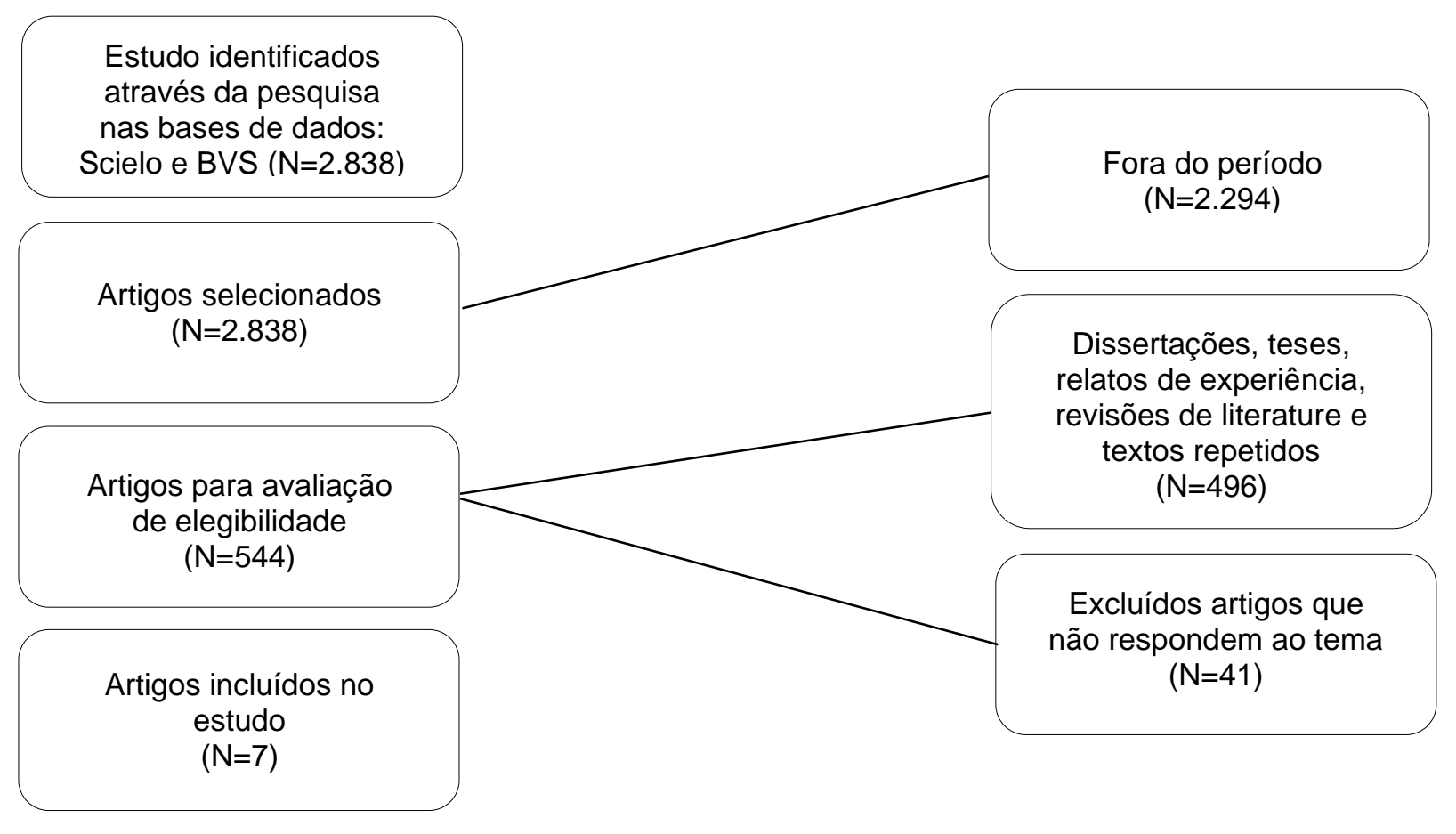

Fonte: Farias RV, et al., 2020.

Posteriormente, foi realizada a leitura na íntegra dos artigos, para compreensão e categorização dos mesmos. Os principais resultados foram interpretados e analisados, emergendo as seguintes categorias: Características maternas e nascimento prematuro; Dados do pré-natal e gestação; Nível de escolaridade e prematuridade.

\section{RESULTADOS}

Após a análise, foram selecionados 7 artigos que envolviam a temática estudada. Os artigos tiveram predominância de realização na região nordeste do Brasil, na modalidade quantitativa, publicados em uma média de 5 anos, em revistas de qualificação A3 e B4, na mesma proporção, pelo Qualis Capes e indexados na base de dados BVS.

Ao realizar a leitura na íntegra dos artigos, foi possível identificar divergências na abordagem da gravidez precoce com a incidência da prematuridade, evidenciando a influência do pré-natal e do nível de escolaridade no desfecho estudado (Quadro 2). 
Quadro 2 - Distribuição dos artigos conforme título, autores, ano, objetivos e principais resultados, 2020.

\begin{tabular}{|c|c|c|c|c|}
\hline № & Autores/Ano & Título & Objetivos & Principais resultados \\
\hline 1 & $\begin{array}{l}\text { ALMEIDA AHV, } \\
\text { et al., } \\
2014\end{array}$ & $\begin{array}{l}\text { Baixo peso ao nascer em } \\
\text { adolescentes e adultas jovens } \\
\text { na Região Nordeste do Brasil }\end{array}$ & $\begin{array}{c}\text { Avaliar a associação entre as características sociodemográficas, } \\
\text { do pré-natal e do parto de mães adolescentes e adultas jovens } \\
\text { com o baixo peso ao nascer, em municípios da Região Nordeste } \\
\text { do Brasil. }\end{array}$ & $\begin{array}{l}\text { As possíveis causas para bebês com baixo peso } \\
\text { foram: número insuficiente de consultas de pré- } \\
\text { natal, primigestação, prematuridade e escolaridade } \\
\text { inadequada. A maior parte das adolescentes } \\
\text { possuíam escolaridade inadequada e número } \\
\text { reduzido de consultas de pré-natal. }\end{array}$ \\
\hline 2 & $\begin{array}{l}\text { SANTOS NLAC, } \\
\text { et al., } \\
2014\end{array}$ & $\begin{array}{l}\text { Gravidez na adolescência: } \\
\text { análise de fatores de risco } \\
\text { para baixo peso, } \\
\text { prematuridade e cesariana }\end{array}$ & $\begin{array}{l}\text { Avaliar a associação entre a gravidez de adolescentes }<16 \text { anos } \\
\text { e a ocorrência de nascidos vivos de baixo peso, prematuridade e } \\
\text { cesariana. }\end{array}$ & $\begin{array}{l}\text { A maioria das gestantes adolescentes estavam na } \\
\text { faixa etária }<16 \text { anos. Estas não realizaram o } \\
\text { número suficiente de consultas de pré-natal. }\end{array}$ \\
\hline 3 & $\begin{array}{l}\text { PEREIRA SSM, } \\
\text { et al., } \\
2018\end{array}$ & $\begin{array}{l}\text { Perfil de Gestantes } \\
\text { Acometidas de Parto } \\
\text { Prematuro em uma } \\
\text { Maternidade Pública }\end{array}$ & $\begin{array}{l}\text { Caracterizar o perfil das gestantes acometidas de parto prematuro } \\
\text { segundo as variáveis: faixa etária, escolaridade, situação } \\
\text { conjugal, ocupação, raça, local de moradia; descrever as } \\
\text { complicações do parto prematuro; e identificar o número de óbitos } \\
\text { por parto prematuro. }\end{array}$ & $\begin{array}{l}\text { Nas gestantes com diagnóstico de parto precoce, } \\
\text { observou-se uma predominância igualitária de } \\
\text { prematuros entre adolescentes e adultas jovens. }\end{array}$ \\
\hline 4 & $\begin{array}{l}\text { CARVALHO JBL, } \\
\text { et al., } \\
2018\end{array}$ & $\begin{array}{l}\text { Condições socioeconômicas } \\
\text { da gestação de bebês } \\
\text { prematuros }\end{array}$ & $\begin{array}{c}\text { Identificar as condições socioeconômicas da gestação de bebês } \\
\text { prematuros. }\end{array}$ & $\begin{array}{c}\text { A maioria das mães que tiveram bebês prematuro } \\
\text { estavam na faixa etária de } 20 \text { a } 35 \text { anos de idade. } \\
\text { As adolescentes possuíam ensino fundamental } \\
\text { incompleto. }\end{array}$ \\
\hline 5 & $\begin{array}{l}\text { RIBEIRO JF, et } \\
\text { al., } \\
2017\end{array}$ & $\begin{array}{l}\text { Complicações obstétricas em } \\
\text { adolescentes atendidas em } \\
\text { uma maternidade pública de } \\
\text { referência }\end{array}$ & $\begin{array}{c}\text { Avaliar as complicações obstétricas em adolescentes atendidas } \\
\text { em uma maternidade pública de referência. }\end{array}$ & $\begin{array}{l}\text { Uma das principais complicações obstétricas entre } \\
\text { as adolescentes foi o parto prematuro. Estas } \\
\text { possuíam alta vulnerabilidade pelo baixo nível } \\
\text { escolar. }\end{array}$ \\
\hline 6 & $\begin{array}{l}\text { SOUZA ML, et } \\
\text { al., } \\
2017\end{array}$ & $\begin{array}{l}\text { Taxa de fertilidade e desfecho } \\
\text { perinatal em gravidez na } \\
\text { adolescência: estudo } \\
\text { retrospectivo populacional }\end{array}$ & $\begin{array}{l}\text { Compreender as características e desfechos relacionados à } \\
\text { saúde de nascidos vivos de mães adolescentes em comparação } \\
\text { com os nascidos vivos de mães adultas, analisando as } \\
\text { tendências de taxas de fertilidade específicas por faixa etária e } \\
\text { associações com os desfechos perinatais selecionados em um } \\
\text { estado brasileiro no período de } 2006 \text { à } 2013 \text {. }\end{array}$ & $\begin{array}{l}\text { A maior parte das adolescentes que tiveram parto } \\
\text { precoce estavam na faixa etária de } 10 \text { a } 14 \text { anos } \\
\text { de idade. Essas não realizaram o pré-natal de } \\
\text { maneira completa e possuíam um risco maior para } \\
\text { desfechos adversos na gestação. }\end{array}$ \\
\hline 7 & $\begin{array}{l}\text { TUON RA, et al., } \\
2016\end{array}$ & $\begin{array}{l}\text { Impacto do monitoramento } \\
\text { telefônico de gestantes na } \\
\text { prevalência da prematuridade } \\
\text { e análise dos fatores de risco } \\
\text { associados em Piracicaba, } \\
\text { São Paulo, Brasil }\end{array}$ & $\begin{array}{l}\text { Verificar o impacto do monitoramento telefônico na prevalência da } \\
\text { prematuridade e identificar os fatores de risco associados ao } \\
\text { parto prematuro em grupo de gestantes monitoradas por meio de } \\
\text { seguimento telefônico mensal, no Município de Piracicaba. }\end{array}$ & $\begin{array}{l}\text { As gestantes que não foram monitoradas } \\
\text { apresentaram uma maior prevalência de } \\
\text { prematuridade em relação às gestantes } \\
\text { monitoradas. Sendo que, a maior parte dos partos } \\
\text { prematuros foram das mães de menor faixa etária } \\
\text { de 10-14 anos. }\end{array}$ \\
\hline
\end{tabular}

Fonte: Farias RV, et al., 2020.

REAS/EJCH | Vol.Sup.n.56 | e3977 | DOI: https://doi.org/10.25248/reas.e3977.2020 Página 5 de 10 


\section{DISCUSSÃO}

A gravidez no extremo inferior da vida reprodutiva tornou-se um grande desafio, pois a gestação, o parto e a maternidade são situações que podem trazer múltiplas consequências emocionais, sociais e econômicas para a saúde da mãe adolescente e do seu filho. Com o aumento do número de gestantes adolescentes surgem grandes obstáculos, por conta dos resultados neonatais adversos encontrados em recém-nascidos de mães dessa faixa etária (ROCHA RCL, 2006).

Diante disso, esse fenômeno tornou-se um problema de saúde pública por ocasionar riscos físicos e psíquicos ao binômio mãe-bebê, gerando alta vulnerabilidade entres estes (NUNES SA, 2010). Considerando que, adolescentes grávidas se apresentam mais susceptíveis e vulneráveis em alguns aspectos, neste artigo foram analisados alguns fatores ligados ao desfecho em estudo, a saber: a condição social, econômica, o nível de escolaridade, o acompanhamento pré-natal adequado, o papel das políticas públicas e da escola como fatores de proteção à saúde materno-infantil, além das condições inerentes à imaturidade biológica de uma gestação precoce. Assim, apresentaremos esses fatores de acordo com as categorias previamente estabelecidas.

\section{Características maternas e nascimento prematuro}

Nessa perspectiva, as complicações da gravidez na adolescência estão relacionadas à múltiplas condições, destacando-se o número reduzido de consultas de pré-natal e o baixo nível de escolaridade (BARALDI ACP, et al., 2007). Além disso, a mãe adolescente possui um maior risco de ter um parto prematuro por sua imaturidade biológica (baixa idade ginecológica), pois a gestação ocorre em um organismo que ainda está em formação física e emocional, e poderá desencadear problemas de crescimento e desenvolvimento, devido a insuficiência uteroplacentária e ao comprometimento da transferência de nutrientes para o bebê, que implicam em complicações na gravidez e problemas no parto (BULHÕES RB, et al., 2018; MARTINEZ EZ, et al., 2011; GALLO JHS, 2011).

Em relação a incidência de recém-nascidos prematuros, Ribeiro JF, et al. (2017) informam que uma das principais complicações obstétricas encontradas entre as adolescentes foi o trabalho de parto prematuro. $\mathrm{Na}$ pesquisa de Pereira SSM, et al. (2018), percebeu-se que os partos prematuros foram na mesma proporção entre adolescentes e adultos jovens. Já no estudo de Carvalho JBL, et al. (2018), a incidência da prematuridade foi maior na faixa etária de 20 a 35 anos de idade. Entretanto, Souza ML, et al. (2017) e Tuon RA, et al. (2016) comunicam que a taxa de nascimento prematuro foi maior na faixa etária entre 10 a 14 anos de idade.

Diante desse cenário, percebe-se a necessidade de investimentos em educação em saúde. Na pesquisa de Carvalho JBL, et al. (2018), os autores ratificam a importância das políticas públicas voltadas ao planejamento reprodutivo, além da acessibilidade aos métodos contraceptivos. No estudo de Souza ML, et al. (2017), nota-se que muitas adolescentes não conseguem ter acesso à métodos contraceptivos, prevenção de doenças e promoção à saúde. Para os mesmos autores, os riscos obstétricos e fetais só serão diminuídos quando houver uma rede de apoio direcionada às adolescentes, que foquem na atenção reprodutiva, prénatal e suporte psicológico e social. Nesse sentido, as ações educativas devem trazer esta temática de forma resolutiva, atentando para o contexto o qual a adolescente está inserida, para que as estratégias traçadas sejam de acordo com a sua realidade (TÔRRES TRF, et al., 2013).

Além disso, e para que haja uma redução no número de gravidez na adolescência, é fundamental que ocorra um diálogo dentro dos lares, entre as adolescentes e seus pais sobre sexualidade, já que ainda é uma dificuldade atual e tem influência direta não somente na gravidez, mas também nas infecções sexualmente transmissíveis. Neste período de desenvolvimento, as adolescentes necessitam de orientações e educação sexual para poderem ter conhecimento a cerca do seu próprio corpo e de sexo seguro, visando a prevenção de agravos à saúde.

Nessa perspectiva, chama-se atenção para que em outros espaços, seja na escola ou nos serviços de saúde, desenvolvam-se momentos de escuta ativa com esses adolescentes, ao mesmo tempo em que sejam incluidas atividades educativas para orientação sobre sexo seguro e/ou gravidez planejada. 
Nesse sentido, Vieira PM e Matsukura TS (2017) ressaltam que o professor, para além de contribuir com a aquisição de novos conhecimentos, encontra-se em um lugar de mediar as informações acessadas tanto nas escolas como em outras fontes, realizando um processo crítico, reflexivo e responsável. Assim, sugerese que nas práticas sejam utilizadas linguagens e metodologias mais ativas, dinâmicas e atuais, nas quais os próprios adolescentes tornem-se participantes ativos e possam também colaborar com a construção das propostas de educação sexual.

Por outro lado, Ribeiro VCS, et al. (2016) referem que no contexto dos serviços de saúde, especialmente na Atenção Básica, o planejamento de ações com vista à gravidez precoce é um problema que precisa ser bem discutido, traçando ações educativas/preventivas por todos os membros da equipe de Estratégia de Saúde da Família, bem como pelos responsáveis pela gestão pública de Saúde.

\section{Dados do pré-natal e gestação}

Quanto às consultas de pré-natal, Souza ML, et al. (2017) informam em seu estudo que as adolescentes realizaram menos consultas de pré-natal quando comparadas às mães com 20 anos ou mais. Essa informação é confirmada por Santos NLAC, et al. (2014), porém esse dado não foi relevante para a ocorrência de prematuridade e baixo peso ao nascer. Contudo, Tuon RA, et al. (2016), relatam que o número de consultas de pré-natal está associado à prematuridade, observando que quanto maior o número de consultas, menor é a porcentagem de partos prematuros.

De acordo com o Ministério da Saúde (BRASIL, 2005), as mulheres devem realizar no mínimo seis consultas de pré-natal, sendo uma no primeiro trimestre, duas no segundo trimestre e três no último trimestre. O acompanhamento gestacional durante o pré-natal é um benefício comprovado pela literatura como fator de proteção para prematuridade, retardo do crescimento intrauterino, baixo peso ao nascer e óbitos maternos e infantis por afecções no período peri e pós-natal (BRASIL, 2020b).

Em pesquisa realizada por Guimarães EAA, et al. (2017), evidenciou-se que o número e a qualidade de consultas de pré-natal são fatores associados à prematuridade, portanto, precisam ser avaliados. Melo EC, et al. (2015) explicam que é importante que as gestantes comecem a realizar o pré-natal o quanto antes, para efetuar os procedimentos e condutas necessárias.

Relatam ainda, que além de identificar a qualidade das consultas é necessário compreender e intervir nos fatores maternos que interferem na continuidade do cuidado, com intuito de evitar complicações obstétricas, como o parto prematuro. Para Almeida AHV, et al. (2014), uma das justificativas para o baixo número de consultas de pré-natal está na consequência da gestação precoce, que não permite que esse atendimento seja continuado. Oliveira AA, et al. (2019) reforçam o argumento de que o número de consultas de pré-natal reduzido está associado com fatores socioeconômicos, familiares e psicológicos, ou seja, fatores de vulnerabilidade.

Ainda, o estudo de Sousa EZT, et al. (2020) aponta que as adolescentes iniciam tardiamente o pré-natal, apenas $12,4 \%$ começaram no primeiro trimestre, conforme preconizado mundialmente como cuidado básico durante a gestação. Soma-se ainda as que não conseguem realizar o número mínimo de consultas, o que potencializa os riscos inerentes a uma gravidez na adolescência; uma vez que a assistência pré-natal de qualidade é importante na prevenção de riscos associados à gestação e ao período neonatal, tornando-se imprescindível o acompanhamento adequado do binômio, a fim de identificar situações de risco precocemente, reduzindo a ocorrência de morbidade e mortalidade (SANTOS NLAC, et al., 2014).

Entretanto, a implementação da Política de Atenção à Saúde do Adolescente no Brasil apresenta vários empecilhos, entre eles a formação de um recurso humano, visto que, as equipes de saúde estão despreparadas e dessensibilizadas para atuar com estes jovens, bem como, observa-se uma baixa frequência dos adolescentes nas Unidades de Saúde que se deve ao fato deles identificarem a ida ao serviço como algo com pouca necessidade, e também porque muitos serviços não possuem trabalhos voltados para esta faixa etária, que possam compreender suas necessidades particulares e seu modo peculiar de procurar e utilizar a assistência à saúde (TÔRRES TRF, et al., 2013). Portanto, faz-se necessário maior investimento em estratégias que facilitem o acesso e a adesão das gestantes aos Serviços de saúde. 


\section{Nível de escolaridade e prematuridade}

Ao se falar no nível de escolaridade, Ribeiro JF, et al. (2017) informam que as adolescentes do estudo apresentaram alta vulnerabilidade, pelo baixo nível de escolaridade. Na pesquisa de Carvalho JBL, et al. (2018), as mães adolescentes na sua maioria possuíam ensino fundamental incompleto, constatando-se que quanto maior o nível de escolaridade, menores os números de adolescentes grávidas.

Corroborando com os achados de Azevedo WF, et al. (2015), onde o nível de escolaridade das mulheres era inversamente proporcional à redução da gravidez na adolescência. Resultado semelhante foi encontrado na pesquisa de Almeida AHV, et al. (2014), onde 60,9\% das puérperas possuíam escolaridade inadequada. Pereira SSM, et al. (2018), afirmam que essa realidade está associada ao menor incentivo social para uma educação adequada.

Em um outro estudo, realizado no Ceará, Goudard MJF, et al. (2016) observaram que 86,7\% das mães com idade inferior a 20 anos, cursou até o Ensino Fundamental, consequentemente possuíam um menor grau de instrução, considerando que, nessa fase do ciclo da vida, os adolescentes ainda estão construindo a carreira profissional, não possuem renda fixa e dependem dos parentes para o próprio sustento e o do filho não planejado.

Nessa perspectiva, a associação entre baixa escolaridade e renda com o desfecho de prematuridade encontrada no presente estudo poderia ser explicada por dificuldades no acesso a alimentos de boa qualidade/quantidade, no acompanhamento pré-natal adequado e, consequentemente, barreira para identificação precoce de morbidades, além de baixa precisão do uso do método Data da Última Menstruação (DUM) em vez do ultrassom obstétrico precoce, mais preciso na análise de desenvolvimento fetal (BLENCOWE H, et al., 2013). Diante disso, fica claro que a desigualdade social é um dos principais determinantes que aumentam à ocorrência da gravidez na adolescência, no sentido de que as classes econômicas menos favorecidas vêm apresentando elevados índices deste evento (TABORDA JÁ, et al.,2014).

Nesse contexto, se faz necessário que exista uma articulação dos serviços de saúde, principalmente da Atenção Básica de Saúde com outros equipamentos sociais frequentados pelos jovens, à exemplo da escola, que é um espaço no qual tem como público adolescentes. Essas ações em saúde articuladas em rede, para além dos muros das unidades do serviço de saúde, considerando sempre o meio social e familiar no qual o adolescente está inserido, ampliam a promoção da educação em saúde sexual e reprodutiva, contribuindo para a diminuição dos casos de gravidez precoce e todas as consequências advindas por meio dela, como a prematuridade. Portanto, podemos observar que a principal forma de prevenção da gravidez precoce é a educação sexual, esta deve ser realizada pela família, escola e pelo profissional de saúde, como pontuado anteriormente; pois, nesta fase nota-se o surgimento de dúvidas e medos entre os adolescentes a respeito da saúde sexual e reprodutiva.

\section{CONSIDERAÇÕES FINAIS}

Partindo do exposto, percebe-se que ainda não há um consenso na literatura científica entre a relação da gestação precoce e a incidência de partos prematuros; porém, a maioria dos estudos retratam uma associação entre essas variáveis, devido a diversos fatores, como: nível de escolaridade, número reduzido de consultas do pré-natal e, principalmente, imaturidade biológica materna. Destaca-se também, a importância da educação como estratégia para comportamento sexual seguro entre os adolescentes, prevenindo a gravidez precoce e doenças sexualmente transmissíveis. Essas condições, em conjunto, influenciarão na qualidade de vida futura da mãe e do recém-nascido, sendo um fator contribuinte para riscos vitais ao nascimento, desenvolvimento e crescimento, podendo refletir até mesmo na mortalidade infantil.

\section{REFERÊNCIAS}

1. ALMEIDA AHV, et al. Baixo peso ao nascer em adolescentes e adultas jovens na Região Nordeste do Brasil. Rev. Bras. Saude Mater. Infant., 2014; 14(3): 279-286. 
2. AZEVEDO WF, et al. Complications in adolescent pregnancy: systematic review of the literature. Einstein, 2015; 13(4): 618-629.

3. BARALDI ACP, et al. Gravidez na adolescência: estudo comparativo das usuárias das maternidades públicas e privadas. Rev. Latino-am Enfermagem, 2007; 15: 799-805.

4. BLENCOWE H, et al. Born Too Soon: the global epidemiology of 15 million preterm births. Reproductive Health, 2013; 10(1): 1-14.

5. BRASIL. Ministério da Saúde. Secretaria de Atenção à Saúde. Departamento de Ações Programáticas Estratégicas. Área Técnica de Saúde da Mulher. Pré-natal e Puerpério: atenção qualificada e humanizada - manual técnico. Brasília: Ministério da Saúde, 2005.

6. BRASIL. Ministério da Saúde. Proteger e cuidar da saúde de adolescentes na atenção básica. Ministério da Saúde, Secretaria de Atenção à Saúde, Departamento de Ações Programáticas e Estratégicas. Brasília: Ministério da Saúde, 2017.

7. BRASIL. Ministério da Saúde. Prevenção de gravidez na adolescência é tema de campanha nacional. Brasília: Ministério da Saúde, 2020a.

8. BRASIL. Ministério da saúde. Quatro de sete indicadores do Previne têm foco na saúde das mulheres. Brasília: Ministério da Saúde, 2020b.

9. BULHÕES RB. Prevalência de Recém Nascidos Pré-Termo de Mães Adolescentes. Id on Line Rev. Mult. Psic., 2018; 11(39): 84-96.

10. BUSSMANN CE, PRETTO B. Relato de experiência: percepção acerca do significado do cortes no corpo do adolescente. Revista Destaques Acadêmicos, Lajeado, 2017 ; 9 (3): 168-185.

11. CARVALHO JBL, et al. Condições socioeconômicas da gestação de bebês prematuros. Rev. de Enferm. UFPE, 2018; 12(2): 386-390.

12. CHANG HH, et al. Preventing preterm births: analysis of trends and potential reductions with interventions in 39 countries with very high human development index. Lancet, 2013; 381(9862): 223-234.

13. CRUZ MS, et al. Perfil Socioeconômico Demográfico, Cultural, Regional e Comportamental da Gravidez na Adolescência no Brasil. Planejamento de políticas públicas, 2016; (46): 243-266.

14. GALLO JHS. Gravidez na adolescência: a idade materna, consequências e repercussões. Rev Bioética, 2011;19(1):179-95.

15. GOUDARD MJF, et al. Inadequacy of the content of prenatal care and associated factors in a cohort in the northeast of Brazil. Ciênc Saúde Coletiva, 2016; 21(4): 1227-1238.

16. GUIMARÃES EAA, et al. Prevalência e fatores associados à prematuridade em Divinópolis, Minas Gerais, 2008-2011: análise do Sistema de Informações sobre Nascidos Vivos. Epidemiologia e Serviços de Saúde, Brasília, 2017; 26(1): 91-98.

17. MARTINEZ EZ, et al. Gravidez na adolescência e características socioeconômicas dos municípios do Estado de São Paulo, Brasil: análise espacial: análise espacial. Cadernos de Saúde Pública, Rio de Janeiro, 2011; 27(5): 855-867.

18. MELO EC, et al. Fatores associados à qualidade do pré-natal: uma abordagem ao nascimento prematuro. Revista da Escola de Enfermagem da Usp., São Paulo, 2015; 49(4): 540-549.

19. MENDES KDS, et al. Revisão integrativa: método de pesquisa para a incorporação de evidências na saúde e na enfermagem. Texto Contexto Enferm., Florianópolis, 2008; 17(4): 758-764.

20. NUNES SA. Problematizando a gravidez na adolescência. Rev. Epos, 2010; 1(1).

21. OLIVEIRA AA, et al. Fatores associados ao nascimento pré-termo: da regressão logística à modelagem com equações estruturais. Cadernos de Saúde Pública, 2019; 35(1): 1-15.

22. ONU. Organização das Nações Unidas. 2020. In: Taxa de gravidez na adolescência no Brasil está acima da média mundial, aponta ONU. Brasília: ONU.

23. PAHO. Pan American Health Organization. 2018. In: Part II: The current status of the health of adolescents and youth in the americas. Washington: PAHO/WHO. E-book.

24. PEREIRA FAF, et al. Desafio das mulheres que foram mães na adolescência quanto a prevenção da gravidez precoce de suas filhas. Revista Unimontes Científica, Montes Claros, 2017; 19(2): 73-86.

25. PEREIRA SSM, et al. Perfil de Gestantes Acometidas de Parto Prematuro em uma Maternidade Pública. Rev. pesqui. cuid. fundam., 2018; 10(3): 758-763.

26. RAMALHO NETO JM, et al. Análise de teorias de enfermagem de Meleis: revisão integrativa. Rev. Bras. Enferm., 2016; 69(1): 162-168.

27. RIBEIRO JF, et al. Complicações obstétricas em adolescentes atendidas em uma maternidade pública de referência. UFPE, 2017; 11(7): 2728-2735.

28. RIBEIRO VCS, et al. Papel do enfermeiro da estratégia de saúde da família na prevenção da gravidez na adolescência. R. enferm. Cent. O. Min., 2016; 6(01): 1957-1975.

29. ROCHA RCL, et al. Prematuridade e baixo peso entre recém-nascidos de adolescentes primíparas. Rev Bras Ginecol Obstet. 2006; 28(9): 530-5.

30. SANTOS NLAC, et al. Gravidez na adolescência: análise de fatores de risco para baixo peso, prematuridade e cesariana. Ciência \& Saúde Coletiva, Rio de Janeiro, 2014; 19(3): 719-726.

31. SBP. Sociedade Brasileira de Pediatria. Guia Prático de Atualização: PREVENÇÃO DA GRAVIDEZ NA ADOLESCÊNCIA. Adolesc. Saúde, Rio de Janeiro, 2019; 11: 1-9.

32. SOUSA EZT, et al. Qualidade de vida de adolescentes grávidas. REAS [Internet], 2020; 12(6): e3161.

33. SOUZA ML, et al. Taxa de fertilidade e desfecho perinatal em gravidez, $m$ na adolescência: estudo retrospectivo populacional. Rev. Latino-americana de Enfermagem, 2017; 25: 1-9. 
34. TABORDA JA, et al. Consequências da gravidez na adolescência para as meninas considerando-se as diferenças socioeconômicas entre elas. Cad. Saúde Colet., Rio de Janeiro, 2014; 22(1): 16-24.

35. TÔRRES TRF, et al. O cuidado de enfermagem na saúde sexual e reprodutiva dos adolescentes. Adolesc Saude, Rio de Janeiro, 2013; 10(Supl. 1): 16-26.

36. TUON RA, et al. Impacto do monitoramento telefônico de gestantes na prevalência da prematuridade e análise dos fatores de risco associados em Piracicaba, São Paulo, Brasil. Cad. Saúde Pública, 2016; 32(7):1-16.

37. VIEIRA PM, MATSUKURA TS. Modelos de educação sexual na escola: concepções e práticas de professores do ensino fundamental da rede pública. Revista Brasileira de Educação, 2017; 22 (69): 453-474.

38. WHO. World Health Organization. 2012. In: Born too soon: the global action report on preterm birth. Geneva: WHO. 